\title{
Bioprosthetic Mesh in Abdominal Wall Reconstruction
}

\author{
Donald P. Baumann, M.D., F.A.C.S. ${ }^{1}$ Charles E. Butler, M.D., F.A.C.S. ${ }^{1}$ \\ ${ }^{1}$ Department of Plastic Surgery, The University of Texas MD Anderson \\ Cancer Center, Houston, Texas \\ Address for correspondence and reprint requests Charles E. Butler, \\ M.D., Department of Plastic Surgery, Unit 1488, The University of \\ Semin Plast Surg 2012;26:18-24. \\ Texas MD Anderson Cancer Center, 1515 Holcombe Blvd., Houston, TX \\ 77030, (e-mail: cbutler@mdanderson.org).
}

Abstract
Keywords
- abdominal wall
- hernia
- surgical mesh
- collagen
reconstructive
surgical
procedures
- adhesions
- biocompatible
materials
- dermis
- bulge
- bioprosthetic and
component
separation

Over the past 20 years, implantable surgical mesh products used for abdominal wall reconstruction have evolved in an attempt to improve mesh-related repairs. Mesh products have evolved from unilaminar synthetic materials to bilaminar synthetic meshes to bioprosthetic mesh products derived from human and animal sources. The continued refinement and development of new mesh materials arises from the clinical need to provide a mesh material that can replicate the host tissue that it is replacing. There has been a longstanding general acceptance of the use of mesh to reinforce a stable ventral hernia repair. However, the addition of a mesh material has improved, but not resolved, the problem of high hernia recurrence following ventral hernia repair. Ventral hernia repair is associated with a 10-year cumulative recurrence rate of $32 \%$ with the use of synthetic mesh materials in small $(<6 \mathrm{~cm})$ elective noncontaminated defects, which is roughly a $50 \%$ reduction compared with suture repair alone. ${ }^{1}$ Despite advances in surgical technique and implantable mesh materials, long-term reported outcomes (including length of stay, need for reoperation, and recurrence rates) have not significantly improved over time. ${ }^{2}$ In addition, the incidences of surgical site occurrences, wound dehiscence, wound infection, seroma, and fascial separation are 10 times higher for elective ventral hernia repair than for other clean general surgery procedures. ${ }^{3-5}$ It has been reported that patients undergoing incisional hernia repair with a history of a previously documented wound infection have a $41 \%$ risk of developing another wound infection, whereas only $12 \%$ of patients without a history of infection developed a wound infection after hernia repair. ${ }^{3}$ These data suggest that ventral hernia repair, unlike other abdominal surgical procedures, has an inherently higher risk for developing wound complications including infection.

Despite significant advances in hernia repair techniques and technologies, recurrence rates following standard ventral hernia repair remain high. Evidence from a randomized,
Issue Theme Abdominal Wall Reconstruction; Guest Editor, Lior Heller, M.D.
Copyright @ 2012 by Thieme Medical Publishers, Inc., 333 Seventh Avenue, New York, NY 10001, USA. Tel: +1(212) 584-4662.
DOI http://dx.doi.org/ 10.1055/s-0032-1302461. ISSN $1535-2188$. 
prospective, controlled trial suggests that $32 \%$ of ventral hernias repaired with synthetic mesh recur within 3 years; the rate approaches $63 \%$ for primary repair alone. ${ }^{1}$ In addition, the risk of hernia recurrence increases with each additional operation. Flum and coworkers reported that $12 \%$ of patients undergoing incisional hernia repair required at least one subsequent reoperation within 5 years; the length of time between reoperations was progressively shorter after each additional repair. The 5-year rates of reoperation were $24 \%$ after the first reoperation, 35\% after the second, and 39\% after the third. ${ }^{2}$ These data underscore the importance of minimizing the risk for subsequent reoperations by employing the best evidence-based approach at the time of the initial hernia repair.

This situation has prompted a search for optimal techniques and mesh materials for use in abdominal wall reconstruction. A mesh material with more favorable properties than traditional mesh could have a major effect on surgical practice and patient outcomes. The ideal mesh material would be nontoxic, avoid chronic inflammation and immune rejection, and resist infection after implantation. It would become completely remodeled into host tissue with mechanical and biologic properties similar to those of the replaced tissue. The mesh would serve as a biologic tissue scaffold and become rapidly revascularized and infiltrated with host cells, to avoid encapsulation and seroma formation. It also needs to maintain its strength and original surface area during remodeling to prevent bulge and/or mesh failure. The mesh must not induce adverse systemic or local reaction or pose a risk of disease transmission. In addition, an optimal mesh material must resist visceral adhesions to limit the risk of bowel obstruction and enterocutaneous fistulization and to facilitate subsequent reoperative laparotomy if needed in the future. The optimal mesh could be implanted into contaminated wounds and tolerate cutaneous exposure without the obligatory requirement of surgical explantation. Unfortunately, this ideal mesh material is not yet available.

\section{Synthetic and Bioprosthetic Mesh Materials}

A wide variety of synthetic mesh products is available for use in abdominal wall reconstruction, including both absorbable and permanent synthetic meshes. However, synthetic mesh has limitations that preclude it from being widely used in abdominal wall reconstruction. For example, absorbable mesh such as polyglactin 910 (Vicryl ${ }^{\circledR}$, Ethicon Inc., Somerville, NJ) can be a useful adjunct in the management of the open abdomen by temporarily containing the viscera, but it becomes degraded and resorbed over time and is associated with an extremely high hernia recurrence rate. ${ }^{6}$

Synthetic meshes are categorized as macroporous, microporous, or composite. ${ }^{7,8}$ Macroporous meshes include monofilament and multiple-filament polypropylene. These materials have large pore sizes that allow for in-growth of scar tissue. When placed in contact with abdominal viscera, macroporous meshes are associated with the formation of bowel adhesions, obstructions, and enterocutaneous fistulae. ${ }^{9,10}$ Therefore, these materials should be avoided or used in combination with omental coverage or antiadhesive barriers when placed in contact with bowel. Microporous meshes, such as expanded polytetrafluoroethylene (ePTFE), have a smaller pore size that does not allow clinically relevant tissue in-growth and may lead to encapsulation, periprosthetic fluid collection, and bacterial overgrowth. Although microporous mesh has a lower affinity for visceral adhesions, it is more susceptible to infection, and when clinical infection occurs surgical explantation is usually required rather than a conservative salvage of the repair. A wide variety of composite materials are now available that combine macroporous mesh (usually polypropylene or polyester) on one side to promote tissue in-growth and an antiadhesive layer on the other (peritoneal) side to reduce risk for adhesions to the mesh repair. Clinical evidence suggests reduced risk of adhesions to composite and coated synthetic meshes compared with traditional synthetic meshes. ${ }^{11-15}$ The relative benefits of these different synthetic meshes with regard to adhesion formation, risk for infection, and outcomes have varied widely in different animal and human clinical studies. ${ }^{9,13,16-19}$ Furthermore, prospective data are lacking regarding the clinical benefits of these prostheses for ventral hernia repair, and no high-level evidence or comparative clinical data are currently available. Finally, lightweight polypropylene mesh with thinner-diameter polypropylene fibers and larger interstices is currently being used in both open and laparoscopic hernia repairs. There are data to suggest good functional outcomes equal or better than those achieved with traditional heavyweight polypropylene synthetic mesh, although definitive long-term comparative studies are lacking. ${ }^{20}$ Despite numerous advances in synthetic mesh technology, the paramount problem still exists: the placement of a persistent foreign body that does not remodel into biologic tissues, the risk of infection, and the management of infected or exposed synthetic mesh.

Bioprosthetic meshes are an equally diverse and expanding class of mesh materials. These are materials derived from human or animal tissue, decellularized and processed to allow implantation into humans. Certain specific characteristics are thought to contribute to the successful use of particular biologic repair materials in the setting of wound contamination or low-grade infection. These mesh properties include an intact, native extracellular matrix and the ability to support tissue regeneration through revascularization and cell repopulation. It has been hypothesized that resistance to infection for biologic repair materials is related to the ingrowth of cells and vasculature structures. ${ }^{21}$ The neovascularization demonstrated in studies of some biologic repair materials may allow these materials to better resist infection when placed in a potentially contaminated field. ${ }^{21}$ The ability of some biologic repair materials to support regeneration is based on animal studies that demonstrated early biologic activity, including cellular infiltration and revascularization. ${ }^{22-25}$ Numerous animal studies have shown that altering the extracellular matrix through suboptimal processing and/ or cross-linking may have a negative effect on host response to the repair material. ${ }^{26-28}$ Resorption and encapsulation have been demonstrated with several biologic repair 
materials in an animal model of abdominal wall repair. ${ }^{26}$ These latter investigators suggested that the lack of integration and tissue regeneration with these materials may account for poor initial wound healing. In one study of abdominal repair following harvest of transverse rectus abdominis musculocutaneous flaps for breast reconstruction, biopsies of the biologic repair material showed cell density, vasculature, and collagen orientation similar to those of normal abdominal fascial tissue. ${ }^{29}$ Another study found that biologic repair material from an irradiated, contaminated abdominal wall repair site that was explanted 14 months after implantation demonstrated remodeling of the biologic repair material, including revascularization and cellular repopulation. ${ }^{30}$ To date, no comparative trials have been completed evaluating different biologic repair materials in incisional hernia repair, and differentiation between products is based on early findings with a limited number of available bioprosthetic mesh materials. Similar animal and clinical studies are awaited for the majority of bioprosthetic mesh materials.

\section{Evolution of the Use of Bioprosthetic Mesh}

The use of autologous deepithelialized and nondeepithelialized skin for inguinal and ventral hernia repair has been described in the literature as an "auto-dermoplasty" for abdominal wall reconstruction. ${ }^{31}$ Therefore, the concept of using biologic tissue for structural repair of abdominal wall defects is not necessarily a new concept. ${ }^{32}$ Bioprosthetic mesh materials are defined as biologic tissue derived from a mammalian source, either allograft or xenograft. The bioprosthetic mesh undergoes processing that completely removes all cellular components while preserving the native extracellular matrix architecture. If the material is processed correctly to be tissue conductive and to maintain the native extracellular matrix microarchitecture, the bioprosthetic mesh material undergoes fibrovascular in-growth with host cell repopulation after implantation and then continues to become remodeled by the host into tissue.

A historical perspective on currently used bioprosthetic mesh materials includes porcine small bowel submucosa (SIS) for bladder reconstruction, human acellular dermal matrix (HADM) for skin and mucosal grafting, and porcine acellular dermal matrix (PADM) for bladder sling suspension and other urogynecologic procedures. ${ }^{33,34}$ Porcine small intestine submucosa was studied in a canine ventral hernia model that showed the SIS extracellular matrix scaffold was replaced by well-organized host tissues including differentiated skeletal muscle. ${ }^{35}$ Human acellular dermal matrix and xenogenic materials such as PADM, bovine acellular dermal matrix (BADM), and bovine pericardium were initially designed for indications other than abdominal wall reconstruction. ${ }^{36-38}$ They were subsequently adopted by surgeons due to the overwhelming need for better mesh properties for abdominal wall reconstruction.

- Table 1 lists some of the commercial bioprosthetic mesh materials available at the time of this article's preparation. These materials are classified by tissue source, animal species, crosslinked versus noncrosslinked, sterilization process, processing details, and storage media. ${ }^{39}$ We do not make any recommendations regarding the choice of specific prosthetic repair materials; however, certain features of synthetic and biologic repair materials should be considered during the selection process. Specific characteristics such as adequate strength, ease of handling during implantation, ability to resist adhesions when placed in contact with the bowel, reduced risk of infection through support for tissue incorporation and revascularization, and early and late mechanical properties are important factors to consider when selecting a bioprosthetic mesh. Differences in mesh processing include the cell extraction and sterilization techniques and whether the mesh is stored in a hydrated state or nonhydrated state within the package. Bioprosthetic mesh materials are also categorized by tissue properties (thickness of the material

Table 1 Bioprosthetic Mesh Materials

\begin{tabular}{|c|c|c|c|c|c|}
\hline Product & Animal Source & $\begin{array}{l}\text { Tissue } \\
\text { Source }\end{array}$ & $\begin{array}{l}\text { Chemically } \\
\text { Crosslinked }\end{array}$ & Sterilization & Manufacturer \\
\hline AlloDerm & Adult human & Dermis & None & None & LifeCell(Branchburg, NJ) \\
\hline Allomax & & & None & Gammairradiation & Bard Davol Inc. (Warwick, RI) \\
\hline Flex HD & & & None & None & Ethicon (Somerville, NJ) \\
\hline Xenmatrix & Adult porcine & Dermis & None & Unknown & Bard Davol Inc. (Warwick, RI) \\
\hline Strattice & & & None & External beamradiation & LifeCell(Branchburg, NJ) \\
\hline Permacol & & & HMDI & Gammairradiation & $\begin{array}{l}\text { Covidien } \\
\text { (Dublin, Ireland) }\end{array}$ \\
\hline Surgisis & & $\begin{array}{l}\text { Small intestine } \\
\text { submucosa }\end{array}$ & None & Ethyleneoxide & $\begin{array}{l}\text { Cook Medical } \\
\text { (Bloomington, IN) }\end{array}$ \\
\hline Veritas & Adult bovine & Pericardium & None & External beamradiation & Synovis (St.Paul, MN) \\
\hline Surgimend & Fetal bovine & Dermis & None & Ethyleneoxide & $\begin{array}{l}\text { TEIBiosciences } \\
\text { (South Boston, MA) }\end{array}$ \\
\hline
\end{tabular}

HMDI, hexamethylene diisocyanate; EDC, 1-ethyl-3-(3-dimethyl-aminopropyl) carbodiimide. 
and size available). Depending on the source tissue (human, bovine, porcine), tissue properties such as thickness, firmness, and size availability will vary.

There is an evolving understanding of the behavior and effect of bioprosthetic mesh in abdominal wall reconstruction, with limited high-level data on the mechanism of action and long-term outcomes, particularly comparative outcomes between products, techniques, and patient selection. Therefore, we as surgeons must make decisions based on what we currently know and what is not as clearly understood. It is clear that bioprosthetic mesh implanted in direct contact with bowel results in fewer adhesions than prosthetic mesh materials. Studies in animal models suggest that certain biologic repair materials can be placed in contact with the bowel. $^{22-24,28}$ Many studies have demonstrated that certain acellular dermal matrices placed directly over the bowel resist visceral adhesions to the repair site in ventral hernia repairs and are markedly less than the adhesions associated with polypropylene repairs. ${ }^{22-24,28}$ Clinical studies have reported good outcomes with some bioprosthetic meshes for incisional hernia repair in high-risk patient groups. In these reports, patients' complications could be managed nonsurgically even when their wounds became infected. ${ }^{40-42}$ Some bioprosthetic meshes have been used successfully to repair large contaminated and/or irradiated abdominal wall defects in patients with cancer when the meshes are placed directly over the bowel. ${ }^{30,43}$ Bioprosthetic mesh materials tolerate placement into contaminated wound environments and are able to undergo vascularization and incorporation, as has been described clinically in the setting of ostomy creation, unplanned bowel surgery, and trauma. ${ }^{4,44}$ This is a critical advantage of bioprosthetic mesh over prosthetic meshes in that the bioprosthetic mesh does not need to be removed in the face of wound contamination or clinically active infection. In addition, bioprosthetic mesh tolerates cutaneous exposure without requiring explantation, and such exposures can usually be managed conservatively with dressing changes. Some biologic repair materials have also demonstrated antimicrobial activity in vitro and in animal models, and the ability of certain biologic prostheses to support revascularization may contribute to clearance of bacteria. ${ }^{45,46}$ A recent study in a rabbit model, for example, found that a human acellular dermal matrix repair material was significantly superior to PTFE in terms of the ability to allow for clearance of Staphylococcus aureus inoculate at the level expected for contamination. ${ }^{47}$

With regard to the processing of the bioprosthetic mesh, the current understanding is that if the native extracellular matrix of the material is preserved, then the mesh is "tissue conductive" and recognized by the host, such that the host remodels the tissue with cellular infiltration, revascularization, and collagen deposition rather than scar tissue and encapsulation. Animal studies found that the early strength of the incorporation into the musculofascial defect edge was similar for bioprosthetic mesh and polypropylene mesh. ${ }^{24}$ Supraphysiologic chemical crosslinking of the collagen within the bioprosthetic mesh significantly reduced cellular and vascular infiltration, reducing the degree of remodeling in animal studies. $^{28,48}$ This results in nearly crosslinked bioprosthetic mesh materials undergoing more of an encapsulation response with surrounding scar tissue rather than actual cellular and vascular infiltration into the mesh, thus limiting the ability to remodel and incorporate.

There is a large gap in the understanding of comparative clinical outcomes between various classes of allograft and xenograft bioprosthetic mesh, as well as for specific commercially available products within each bioprosthetic mesh class. Definitive studies of evidence-based indications and contraindications based on high-level data such as long-term clinical prospective randomized trials are yet to be completed. In addition, studies of long-term comparative outcomes of bioprosthetic mesh compared with other synthetic mesh options have yet to be performed. This is important for specific indications where both mesh types (bioprosthetic or synthetic) can be used. One of the potential shortcomings of bioprosthetic mesh is the potential for bulge and laxity; this has been demonstrated with the use of human acellular dermal matrix with varied but clinically important high rates of bulge and laxity. ${ }^{30,49}$ It is unclear how the xenograft bioprosthetic meshes will perform over the long term when evaluated for recurrent hernia or bulge formation. An additional factor that merits discussion is the cost-effectiveness of bioprosthetic mesh compared with synthetic mesh alternatives. Studies are needed on the economic impact of using bioprosthetic mesh for various indications, ranging from the most complicated cases (where it may very well be the only option) to more routine cases (where other mesh types may be options). This will require large multicenter trials of various patient populations, defect types, and abdominal wall repair techniques. More data need to be generated to establish functional outcomes, patient selection, and economic value.

\section{Current Indications for Utilization of Bioprosthetic Mesh}

The following indications are based largely on existing animal data, short-term, low-level evidence from clinical studies, case reports, and surgeons' experiences with extensive use of bioprosthetic and synthetic mesh. These indications are general and are based on our opinions and the existing low-level evidence. ${ }^{50}$

1. Contaminated wound (existing wound infection, adjacent ostomy, planned or inadvertent disruption of the gastrointestinal continuity, enterocutaneous fistula)

2. Complex repair in a patient at high risk for development of wound-healing problems such as subcutaneous infection, persistent seroma, skin dehiscence, and/or need for reoperation

3. Planned exposed mesh or high likelihood of a cutaneous exposure (open abdominal wound closure with a bridging repair or unreliable skin coverage with risk of skin dehiscence)

4. Unavoidable direct placement of implantable mesh over bowel and/or other abdominal viscera

5. Planned or high likelihood of the patient requiring a future laparotomy through the repair site 


\section{Technical Considerations To Optimize Outcomes With Bioprosthetic Mesh Reconstruction of the Abdominal Wall}

The overall principles of abdominal wall reconstruction include optimization of the patient, preparation of the wound, centralization and reapproximation of the rectus muscles along the midline when possible, and the use of appropriate prosthetic or bioprosthetic material to reinforce the closure. One key element of the inset of bioprosthetic mesh is to place the mesh in an inlay (intraperitoneal), preperitoneal, or retrorectus position under appropriate physiologic tension. Onlay bioprosthetic mesh placement has been used successfully and is an option when the musculofascia can be primarily closed at the midline. Onlay mesh may be preferred in some situations, such as a hostile abdomen in which adequate lysis of adhesions is unsafe or impossible to expose the undersurface of the musculofascial edges. Physiologic tension represents the resting tension of the abdominal wall when the patient is awake. This is in contradistinction to a tensionfree repair, popularized with autologous inguinal hernia repairs in the past. Mesh inset under physiologic tension facilitates and stimulates appropriate collagen remodeling to facilitate mechanical strength and potentially reduce the risk of material bulge and laxity. The edges of the bioprosthetic mesh should overlap the undersurface of the musculofascial edge by at least 3 to $5 \mathrm{~cm}$ to allow for remodeling fibrovascular incorporation. This method of bioprosthetic mesh inlay takes advantage of the mesh's remodeling mechanism, in contrast to simple scarring and encapsulation with nontissue-conductive meshes. ${ }^{23,28,51}$ This will maximize the ultimate tensile strength of the junction between the bioprosthetic mesh and the musculofascia.

The anatomic plane of the inlay bioprosthetic mesh inset has direct implications on the degree of incorporation at the mesh-musculofascial interface. When possible, it is preferable to avoid insetting the bioprosthetic mesh in direct contact with the peritoneum or preperitoneal fat. To avoid this, the senior author (CEB) prefers to dissect the preperitoneal fat pad away from the posterior rectus sheath so that the mesh is placed in direct continuity with the posterior sheath fascia. This improves the mechanical strength at the mesh-musculofascial interface rather than suturing the mesh to the preperitoneal fat and peritoneal surface. In addition, the preperitoneal flap can be positioned dorsally to the bioprosthetic mesh suture line to further limit adhesion in the suture inset areas. Alternatively, the retrorectus plane presents another good option when the bioprosthetic mesh is sutured to the semilunar line from between the rectus muscle and the posterior rectus sheath. Onlay mesh placement is technically easier but has several significant drawbacks; we do not recommend it as a primary option. The limitations of the onlay repair include the fact that if a reinforced repair is going to be performed, one generally must be able to close the fascia first; this is not possible in many of the cases where there are large defects and the inset of the material as an inlay actually helps offset the tension to close the fascial defect. Thus, an inlay mesh placement facilitates primary fascial closure, whereas onlay mesh placement is performed after primary fascial closure is achieved. In addition, the seroma rate is at least theoretically higher when the bioprosthetic mesh is placed in the subcutaneous plane. Although there may be some biomechanical advantages to an inlay repair compared with an onlay repair, there may be some situations in which an onlay repair may be the only safe option, as is the case when it is impractical to reenter a hostile abdomen.

The bioprosthetic mesh inlay repair is reinforced in a second layer of primary fascial closure over bioprosthetic mesh whenever technically feasible. This dual layer repair is preferred to a bridging interposition repair. Centralization of the rectus abdominis muscle complexes reduces the fascial defect and facilitates primary fascial closure. Primary fascial coverage also allows for complete apposition of bioprosthetic mesh to the surrounding musculofascial defect edge, eliminates bioprosthetic mesh exposure to the subcutaneous fat, and may limit seroma formation. In circumstances in which primary fascial closure is not attainable, a bridging repair is performed. This is performed as a dual circumferential inlay technique ${ }^{43}$ that allows two concentric suture lines to affix the bioprosthetic mesh directly to the musculofascia. Creating direct opposition of the bioprosthetic mesh to the undersurface of the fascial defect itself prevents any fluid collections from occurring between the two layers, which can result in lack of or delay in fibrovascular incorporation and remodeling. In addition, closed suction drains are placed between the musculofascia and the bioprosthetic mesh to prevent any potential fluid collections from developing at the mesh-musculofascial interface.

An adjunct surgical technique to improve outcomes in abdominal wall reconstruction is to combine bioprosthetic mesh inlay repair with component separation. Component separation has the ability to medialize the rectus complexes and reduce the defect size, with the ultimate goal of allowing a primary fascial closure over the inlay bioprosthetic mesh and therefore reinforce a repair. Component separation also will reduce the subsequent tension on the midline, reducing the fascial incision closure and/or musculofascia to implant incision tension. This is accomplished by separating the external oblique aponeurosis and delaminating the external oblique muscle from the internal oblique muscle interface. This results in an offloading of the bilateral superolateral vector distraction of the external oblique muscle on the central wound closure.

At times, unfavorable wound-healing scenarios will be encountered, with wound infection, dehiscence, or breakdown of overlying skin flaps leading to exposure of the bioprosthetic mesh. The bioprosthetic mesh's tolerances of bacterial contamination and exposure allow an acute opening due to wound separation to be reclosed over drains after clearing any infection and ensuring there is adequate skin laxity for closure. Small defects can be left open to heal by secondary intension with standard saline-soaked dressing changes or negative pressure wound therapy (NPWT) devices. NPWT is a useful adjunct in the management of exposed 
bioprosthetic mesh. The goal of NPWT is to prevent desiccation and dehydration of the bioprosthetic material. NPWT can be used to develop a revascularized mesh granulation bed suitable for skin graft coverage or serve as a temporizing measure to facilitate a delayed primary closure or flap coverage as the clinical circumstances dictate. The use of nonadherent barrier dressing materials between the NPWT foam and bioprosthetic mesh prevents trauma to the bioprosthetic mesh and prevents foam retention on the bioprosthetic mesh. Various materials can be used, such as wide-mesh petroleum-impregnated gauze or perforated silicone dressings; alternatively, the use of microporous foam (such as polyvinyl alcohol foam) directly over the bioprosthetic mesh is an option. A skin graft can be applied onto granulated bioprosthetic mesh. It is unknown exactly what clinical indicators are reliable for determining when a skin graft will survive over the bioprosthetic mesh. In our experience, this typically does not require complete granulation tissue over the material but rather the appearance of buds of granulation tissue to the surface of the material through the existing adnexal structures (hair follicle and sweat gland channels). If the defect is large with exposed bioprosthetic mesh at the base, the best choice is generally autologous skin flap tissue, either locally advanced from the abdomen, rotation advancement flap, pedicled regional flap, or free flap.

\section{Conclusion}

Bioprosthetic mesh products have several potential advantages over other synthetic permanent materials in select clinical situations. Indications for implantation of bioprosthetic mesh in abdominal wall reconstruction include contaminated wounds, complex repairs at high risk for developing wound-healing problems, high likelihood of a cutaneous exposure, and unavoidable direct placement of mesh over bowel. Further studies will be important for comparing long-term outcome and cost factors to better define the role, indications, and contraindications for the use of bioprosthetic mesh in abdominal wall reconstruction.

\section{Financial Disclosures}

Dr. Butler has served as a consultant for LifeCell Corporation in Branchburg, New Jersey.

\section{References}

1 Burger JW, Luijendijk RW, Hop WC, Halm JA, Verdaasdonk EG, Jeekel J. Long-term follow-up of a randomized controlled trial of suture versus mesh repair of incisional hernia. Ann Surg 2004;240 (4):578-583, discussion 583-585

2 Flum DR, Horvath K, Koepsell T. Have outcomes of incisional hernia repair improved with time? A population-based analysis. Ann Surg 2003;237(1):129-135

3 Houck JP, Rypins EB, Sarfeh IJ, Juler GL, Shimoda KJ. Repair of incisional hernia. Surg Gynecol Obstet 1989;169(5):397-399

4 Dunne JR, Malone DL, Tracy JK, Napolitano LM. Abdominal wall hernias: risk factors for infection and resource utilization. J Surg Res 2003;111(1):78-84
5 Luijendijk RW, Hop WC, van den Tol MP, et al. A comparison of suture repair with mesh repair for incisional hernia. N Engl J Med 2000;343(6):392-398

6 Pans A, Elen P, Dewé W, Desaive C. Long-term results of polyglactin mesh for the prevention of incisional hernias in obese patients. World J Surg 1998;22(5):479-482, discussion 482-483

7 den Hartog D, Dur AH, Tuinebreijer WE, Kreis RW. Open surgical procedures for incisional hernias. Cochrane Database Syst Rev 2008(3):CD006438

8 Korenkov M, Paul A, Sauerland S, et al. Classification and surgical treatment of incisional hernia. Results of an experts' meeting. Langenbecks Arch Surg 2001;386(1):65-73

9 Harrell AG, Novitsky YW, Peindl RD, et al. Prospective evaluation of adhesion formation and shrinkage of intra-abdominal prosthetics in a rabbit model. Am Surg 2006;72(9):808-813, discussion 813-814

10 Novitsky YW, Harrell AG, Cristiano JA, et al. Comparative evaluation of adhesion formation, strength of ingrowth, and textile properties of prosthetic meshes after long-term intra-abdominal implantation in a rabbit. J Surg Res 2007;140(1):6-11

11 Bellon JM, Rodriguez M, Garcia-Honduvilla N, Gomez-Gil V, Pascual G, Bujan J. Postimplant behavior of lightweight polypropylene meshes in an experimental model of abdominal hernia. J Invest Surg 2008;21(5):280-287

12 Emans PJ, Schreinemacher MH, Gijbels MJ, et al. Polypropylene meshes to prevent abdominal herniation. Can stable coatings prevent adhesions in the long term? Ann Biomed Eng 2009;37 (2):410-418

13 van 't Riet M, de Vos van Steenwijk PJ, Bonthuis F, et al. Prevention of adhesion to prosthetic mesh: comparison of different barriers using an incisional hernia model. Ann Surg 2003;237(1):123-128

14 Schug-Pass C, Sommerer F, Tannapfel A, Lippert H, Köckerling F. The use of composite meshes in laparoscopic repair of abdominal wall hernias: are there differences in biocompatibility?: experimental results obtained in a laparoscopic porcine model Surg Endosc 2009;23(3):487-495

15 Pierce RA, Perrone JM, Nimeri A, et al. 120-day comparative analysis of adhesion grade and quantity, mesh contraction, and tissue response to a novel omega-3 fatty acid bioabsorbable barrier macroporous mesh after intraperitoneal placement. Surg Innov 2009;16(1):46-54

16 Harrell AG, Novitsky YW, Kercher KW, et al. In vitro infectability of prosthetic mesh by methicillin-resistant Staphylococcus aureus. Hernia 2006;10(2):120-124

17 Schreinemacher MH, Emans PJ, Gijbels MJ, Greve JW, Beets GL, Bouvy ND. Degradation of mesh coatings and intraperitoneal adhesion formation in an experimental model. $\mathrm{Br} \mathrm{J}$ Surg 2009;96(3):305-313

18 de Vries Reilingh TS, van Goor H, Koppe MJ, Bodegom ME, Hendriks T, Bleichrodt RP. Interposition of polyglactin mesh does not prevent adhesion formation between viscera and polypropylene mesh. J Surg Res 2007;140(1):27-30

19 Burger JW, Halm JA, Wijsmuller AR, ten Raa S, Jeekel J. Evaluation of new prosthetic meshes for ventral hernia repair. Surg Endosc 2006;20(8):1320-1325

20 Cobb WS, Kercher KW, Heniford BT. The argument for lightweight polypropylene mesh in hernia repair. Surg Innov 2005;12(1): 63-69

21 Holton LH III, Kim D, Silverman RP, Rodriguez ED, Singh N, Goldberg NH. Human acellular dermal matrix for repair of abdominal wall defects: review of clinical experience and experimental data.J Long Term Eff Med Implants 2005;15(5):547-558

22 Butler CE, Prieto VG. Reduction of adhesions with composite AlloDerm/polypropylene mesh implants for abdominal wall reconstruction. Plast Reconstr Surg 2004;114(2):464-473

23 Burns NK, Jaffari MV, Rios CN, Mathur AB, Butler CE. Non-crosslinked porcine acellular dermal matrices for abdominal wall reconstruction. Plast Reconstr Surg 2010;125(1):167-176 
24 Gobin AS, Butler CE, Mathur AB. Repair and regeneration of the abdominal wall musculofascial defect using silk fibroin-chitosan blend. Tissue Eng 2006;12(12):3383-3394

25 Holton LH III, Chung T, Silverman RP, et al. Comparison of acellular dermal matrix and synthetic mesh for lateral chest wall reconstruction in a rabbit model. Plast Reconstr Surg 2007;119 (4):1238-1246

26 Sandor M, Xu H, Connor J, et al. Host response to implanted porcine-derived biologic materials in a primate model of abdominal wall repair. Tissue Eng Part A 2008;14(12):2021-2031

27 Jarman-Smith ML, Bodamyali T, Stevens C, Howell JA, Horrocks M, Chaudhuri JB. Porcine collagen crosslinking, degradation and its capability for fibroblast adhesion and proliferation. J Mater Sci Mater Med 2004;15(8):925-932

28 Butler CE, Burns NK, Campbell KT, Mathur AB, Jaffari MV, Rios CN. Comparison of cross-linked and non-cross-linked porcine acellular dermal matrices for ventral hernia repair. J Am Coll Surg 2010;211(3):368-376

29 Glasberg SB, D’Amico RA. Use of regenerative human acellular tissue (AlloDerm) to reconstruct the abdominal wall following pedicle TRAM flap breast reconstruction surgery. Plast Reconstr Surg 2006;118(1):8-15

30 Nemeth NL, Butler CE. Complex torso reconstruction with human acellular dermal matrix: long-term clinical follow-up. Plast Reconstr Surg 2009;123(1):192-196

31 Hagström P, Nylén B. Repair of incisional hernias and defects in the anterior abdominal wall using dermal grafts. Case report. Scand J Plast Reconstr Surg 1976;10(2):157-158

32 Fogh-Andersen P. Repair of monstrous ventral hernias with buried dermis or whole skin grafts. Acta Chir Scand 1963;126;466-473

33 Jarvis GJ, Fowlie A. Clinical and urodynamic assessment of the porcine dermis bladder sling in the treatment of genuine stress incontinence. Br J Obstet Gynaecol 1985;92(11):1189-1191

34 Kim JY, Bullocks JM, Basu CB, et al. Dermal composite flaps reconstructed from acellular dermis: a novel method of neourethral reconstruction. Plast Reconstr Surg 2005;115(7):96e-100e

35 Badylak S, Kokini K, Tullius B, Simmons-Byrd A, Morff R. Morphologic study of small intestinal submucosa as a body wall repair device. J Surg Res 2002;103(2):190-202

36 Sheridan RL, Choucair RJ. Acellular allogenic dermis does not hinder initial engraftment in burn wound resurfacing and reconstruction. J Burn Care Rehabil 1997;18(6):496-499

37 Tufaro AP, Buck DW II, Fischer AC. The use of artificial dermis in the reconstruction of oncologic surgical defects. Plast Reconstr Surg 2007;120(3):638-646

38 Sullivan SA, Dailey RA. Graft contraction: a comparison of acellular dermis versus hard palate mucosa in lower eyelid surgery. Ophthal Plast Reconstr Surg 2003;19(1):14-24
39 Bellows CF, Alder A, Helton WS. Abdominal wall reconstruction using biological tissue grafts: present status and future opportunities. Expert Rev Med Devices 2006;3(5):657-675

$40 \mathrm{Kim} \mathrm{H}$, Bruen K, Vargo D. Acellular dermal matrix in the management of high-risk abdominal wall defects. Am J Surg 2006;192 (6):705-709

41 Diaz JJ Jr, Guy J, Berkes MB, Guillamondegui O, Miller RS. Acellular dermal allograft for ventral hernia repair in the compromised surgical field. Am Surg 2006;72(12):1181-1187, discussion 1187-1188

42 Patton JH Jr, Berry S, Kralovich KA. Use of human acellular dermal matrix in complex and contaminated abdominal wall reconstructions. Am J Surg 2007;193(3):360-363, discussion 363

43 Butler CE, Langstein HN, Kronowitz SJ. Pelvic, abdominal, and chest wall reconstruction with AlloDerm in patients at increased risk for mesh-related complications. Plast Reconstr Surg 2005;116 (5):1263-1275, discussion 1276-1277

44 Campbell A, Chang M, Fabian T, et al; Open Abdomen Advisory Panel. Management of the open abdomen: from initial operation to definitive closure. Am Surg 2009;75(11, Suppl):S1S22

45 Sarikaya A, Record R, Wu CC, Tullius B, Badylak S, Ladisch M. Antimicrobial activity associated with extracellular matrices. Tissue Eng 2002;8(1):63-71

46 Badylak SF, Coffey AC, Lantz GC, Tacker WA, Geddes LA. Comparison of the resistance to infection of intestinal submucosa arterial autografts versus polytetrafluoroethylene arterial prostheses in a dog model. J Vasc Surg 1994;19(3):465-472

47 Milburn ML, Holton LH, Chung TL, et al. Acellular dermal matrix compared with synthetic implant material for repair of ventral hernia in the setting of peri-operative Staphylococcus aureus implant contamination: a rabbit model. Surg Infect (Larchmt) 2008;9(4):433-442

48 Orenstein SB, Qiao Y, Klueh U, Kreutzer DL, Novitsky YW. Activation of human mononuclear cells by porcine biologic meshes in vitro. Hernia 2010;14(4):401-407

49 Jin J, Rosen MJ, Blatnik J, et al. Use of acellular dermal matrix for complicated ventral hernia repair: does technique affect outcomes? J Am Coll Surg 2007;205(5):654-660

50 Breuing K, Butler CE, Ferzoco S, et al; Ventral Hernia Working Group. Incisional ventral hernias: review of the literature and recommendations regarding the grading and technique of repair. Surgery 2010;148(3):544-558

51 Sandor M, Xu H, Connor J, et al. Host response to implanted porcine-derived biologic materials in a primate model of abdominal wall repair. Tissue Eng Part A 2008;14(12):20212031 